\title{
The Immediate Effect of Anterior Pelvic Tilt Taping on Pelvic Inclination
}

\author{
Jung-HoOn LeE ${ }^{1)}$, Won-Gyu YoO ${ }^{2)}$, GAK HWANG-Bo ${ }^{3)}$ \\ 1) Department of Physical Therapy, The Graduate School, Inje University \\ ${ }^{2)}$ Department of Physical Therapy, College of Biomedical Science and Engineering, Inje University: \\ 607 Obangdong, Gimhae, Gyeongsangnam-do, 621-749 Republic of Korea. \\ TEL: +82 55-320-3994,FAX: +82 55-329-1678,E-mail: won7y@inje.ac.kr \\ 3) Department of Physical Therapy, College of Rehabilitation Science, Daegu University
}

\begin{abstract}
Purpose] The purpose of this study was to investigate the effect of anterior pelvic tilt taping (APTT) on the anterior inclination of the pelvis when Kinesio tape (KT) was applied to the erector spinae and the internal oblique, which are involved in anterior pelvic tilt. [Subjects] Forty asymptomatic adults (twenty-three males and seventeen females) agreed to participate in this experiment. [Methods] First, the pelvic inclination of both sides was measured with a palpation meter (PALM), as the subjects leaned the anterior aspect of their thighs against a stabilizing table, in an upright standing posture. After applying KT to the erector spinae and the internal oblique, the anterior inclination of the pelvis of both sides was measured again. [Results] The anterior inclination of the pelvis of both sides significantly increased when APTT was applied to the erector spinae and the internal oblique in both males and females. [Conclusion] These results suggest that APTT with KT, applied to the muscles involved in anterior inclination, for the purpose of tilting the posteriorly tilted pelvic anteriorly, may change pelvic inclination.
\end{abstract}

Key words: Anterior pelvic tilt taping, Kinesio tape, Pelvic inclination

(This article was submitted Aug. 18, 2010, and was accepted Sep. 10, 2010)

\section{INTRODUCTION}

Lumbar lordosis is the compensatory curvature that directly delivers the center of gravity of the head, neck, and trunk to both the legs, distributing the body weight and promoting posture ${ }^{1}$. There is a close relationship between changes in pelvic tilt and changes in lumbar lordosis measured in the standing position ${ }^{2}$. Such changes in lordosis alter the location of the nucleus pulposus within in vertebral discs, as well as the diameter of the intervertebral foramina $^{3)}$. The most important aspect of anterior pelvic tilt is that it increases lumbar lordosis, which can optimize the alignment of the entire spine ${ }^{3)}$. However, abnormal positions strain ligaments and muscles, and indirectly affect the curvature of the lumbar spine ${ }^{4}$. In many studies, the relationship between changes in lumbar lordosis and back pain has been investigated ${ }^{5}$. Some studies have reported that patients with chronic low back pain experience loss of lumbar lordosis ${ }^{6,7)}$ and they demonstrate overly posterior pelvic tilt compared with healthy individuals ${ }^{8}$. Posterior pelvic tilt reduces lordosis by flexing the lumbar spine, which causes posterior movement of the nucleus pulposus and an increase in the intervertebral foramina diameter ${ }^{3)}$. When lumbar lordosis is decreased, imbalance in the center of gravity of the anterior to the sacrum may be created ${ }^{9}$.
There are many manipulation methods available for pelvic treatment ${ }^{10)}$, but the effects of these manipulations are still controversial. In particular, one study concerning the effect of manipulation on the position between the ilium and the sacrum found that manipulation-mobilization, as evaluated by Roentgen stereophotogrammetric analysis (RSA) in the standing position, failed to alter the position between the ilium and the sacrum ${ }^{11)}$.

The use of KT is gradually increasing in the clinical field, not only for reducing pain but also for normalizing muscular function $^{12)}$. In a study on traumatic patella dislocation, KT showed beneficial effects, including pain reduction, quadriceps strengthening, and increased weight bearing stability ${ }^{13)}$. Many studies have shown that leuko tape, a nonelastic tape, can be used to reduce pain and improve function e.g., there are cases of patients with patellofemoral syndrome showing improved patellar location ${ }^{14,15}$, and cases in which the humeral heads of shoulder impingement patients were relocated ${ }^{16)}$. However, no researchers have yet studied the repositioning of pelvic inclination using KT. Thus, the purpose of this study was to investigate the immediate effect of anterior pelvic tilt taping (APTT) on the anterior inclination of the pelvis after application of KT to the erector spinae and internal oblique on both sides of asymptomatic adults. 


\section{SUBJECTS AND METHODS}

The subjects of this study were 23 males [age: $23.8 \pm 1.3$ years (mean $\pm \mathrm{SD}$ ); height: $175.4 \pm 4.9 \mathrm{~cm}$; body weight $69.7 \pm 8.1 \mathrm{~kg}$ ] and 17 females [age: $21.4 \pm 1.1$ years (mean $\pm \mathrm{SD}$ ); height: $161.1 \pm 3.6 \mathrm{~cm}$; body weight $50.3 \pm 6.4 \mathrm{~kg}$ ]. The subjects had no limitations in the ranges of movement of the pelvis or spine, and no orthopedic disabilities (such as deformity, fracture, or arthritis of the pelvis or spine). The subjects read and signed an informed-consent document that was approved by the Human Ethics Committee of the Faculty of Health Sciences at Inje University prior to their participation.

The palpation meter, PALM, (Performance Attainment Associates, St. Paul, MN, USA) consists of an inclinometer and two caliper arms. It is a reliable and cost-effective instrument used to calculate height discrepancies between landmarks. The validity estimates of PALM measurements are excellent, when compared with radiograph measurements ${ }^{17}$. Some studies have used PALM to measure static innominate rotation of the ipsilateral anterior superior iliac spine (ASIS) and posterior superior iliac spine (PSIS $)^{18,19)}$. The intra-test reliability of the PALM is 0.90 , and the inter-test reliability is 0.85 .

The subjects wore non-restrictive clothing and removed their shoes during the measurement. They spread their feet (about $10-12 \mathrm{~cm}$ ) and stood in a relaxed posture ${ }^{20)}$. They stood in an upright position, leaning the anterior aspect of the thighs against a stabilizing table ${ }^{20}$. The investigator palpated the prominence of the ipsilateral ASIS and PSIS and marked them with a pen. The sagittal plane rotation of the innominate bone was measured with the caliper tips of the PALM in contact with the ipsilateral ASIS and PSIS. While the patient remained in the the same position, KT was stretched approximately 15 to $25 \%$, (as recommended by $\mathrm{Kase}^{21)}$ ) and applied to the erector spinae and the internal oblique on both sides by a physical therapist with expertise in KT. For the erector spinae involved in anterior pelvic tilt, KT was applied from the bottom side of the iliac crest to the inferior borders of the angles of the lower 6-7 ribs along the direction of the muscle fibers to render a mechanical effect. For the internal oblique, KT was applied from the bottom side of the middle $1 / 3$ of the intermediate line of the iliac crest to the inferior borders of the 10th through 12th ribs and the linea alba by means of an aponeurosis along the iliac crest intermediate line, and obliquely medially and upwards to render a mechanical effect. In addition, to minimize possible error, the change in pelvic inclination between ASIS and PSIS was measured with PALM by the same individual. An anterior pelvic tilt angle is shown as a positive $(+)$ value, and a posterior pelvic tilt angle as a negative $(-)$ value.

SPSS software (version 14.0, SPSS, Chicago, IL, USA) was used to examine the change in pelvic inclination after the anterior pelvic tilt taping. A paired t-test was used to find the difference between the measurements before and after APTT, with a significance level of $\mathrm{p}<0.01$.
Table 1. Comparison of the anterior pelvic tilt angle before and after APTT $(\mathrm{n}=40)$

\begin{tabular}{lcc}
\hline \multirow{2}{*}{ Inclination } & \multicolumn{2}{c}{ Mean $\pm \mathrm{SD}\left({ }^{\circ}\right)$} \\
\cline { 2 - 3 } & Without APTT & With APTT \\
\hline R. ASIS-PSIS Tilt Angle (M) & $8.17 \pm 2.43$ & $9.58 \pm 2.43^{*}$ \\
L. ASIS-PSIS Tilt Angle (M) & $7.62 \pm 1.86$ & $9.63 \pm 1.70^{*}$ \\
R. ASIS-PSIS Tilt Angle (F) & $7.72 \pm 2.08$ & $10.32 \pm 2.80^{*}$ \\
L. ASIS-PSIS Tilt Angle (F) & $8.06 \pm 2.46$ & $10.28 \pm 2.70^{*}$ \\
\hline
\end{tabular}

R: Right, L: Left, ASIS: anterior superior iliac spine, PSIS: posterior superior iliac spine, M: Male, F: Female, APTT: Anterior Pelvic Tilt Taping, *: $\mathrm{p}<0.01$.

\section{RESULTS}

The anterior pelvic tilt of the male participants was significantly increased after APTT application $(\mathrm{p}<0.01)$. The left pelvic inclination of the males was $7.62 \pm 1.86^{\circ}$ before APTT application and $9.63 \pm 1.70^{\circ}$ after. The right pelvic inclination also increased from $8.17 \pm 2.43^{\circ}$ before to $9.58 \pm 2.43^{\circ}$ after taping (Table 1 ). The anterior pelvic tilt of the female participants also significantly increased after APTT application $(\mathrm{p}<0.01)$. The left pelvic inclination of the females was $8.06 \pm 2.46^{\circ}$ before APTT application and $10.28 \pm 2.70^{\circ}$ after. The right pelvic inclination also increased from $7.72 \pm 2.08^{\circ}$ before to $10.32 \pm 2.80^{\circ}$ after (Table 1).

\section{DISCUSSION}

This study was conducted to investigate the immediate effect of APTT on the anterior inclination of the pelvis by applying KT to the erector spinae and internal oblique. For both male and female participants, APTT increased the anterior inclination of the pelvis on both sides.

In a study where pelvic taping was applied to a female collegiate tennis player whose ilium showed excessive anterior tilt, leuko tape (a non-elastic tape) was applied in the direction from ASIS to PSIS to limit anterior pelvic tilt, and the stability of the sacroiliac joint increased ${ }^{22}$. The erector spinae and internal oblique, to which $\mathrm{KT}$ was applied in this study, are the muscles that support and stabilize the sacroiliac joint ${ }^{3)}$. Since the erector spinae is attached to the sacrum and the pelvis, it may increase lumbar lordosis by causing an anterior tilt of the pelvis ${ }^{3)}$. In addition, the internal oblique upper anterior fiber tilts the pelvis forward when the thorax is fixed ${ }^{23)}$. It has been reported that cutaneous stimulation induces reflex contractions of the muscles underlying the skin and that this cutaneous stimulation can reduce the threshold value of the recruitment of motor units of those muscles ${ }^{24)}$. In other words, KT application may stimulate cutaneous mechanoreceptors ${ }^{25)}$, strengthen weakened muscles, and normalize muscle functions ${ }^{26}$. Moreover, KT may affect the muscle and myofascia functions by increasing lymph circulation at the point of application ${ }^{26,27)}$. KT may also improve the excitability of the muscles beneath the skin where it is applied ${ }^{27)}$, suggesting that KT may play a role in 
the anterior tilt of the pelvis by promoting the functions of the erector spinae and the internal oblique. Furthermore, it has been verified that the mechanical benefits of patellar taping achieved with leuko tape (a non-elastic tape) are also evident when KT (a elastic tape) is applied to the muscles surrounding the pelvis. Taking advantage of these benefits, APTT may increase the lumbar lordosis angle of persons who have a flat-back posture. Clinically, it has been suggested that maintenance of normal lumbar lordosis is related to the prevention of spinal disorders ${ }^{28)}$. Maintaining the natural lordotic posture was the fundamental principle that McKenzie used to treat herniated disc patients ${ }^{3)}$. An increase in lumbar extension reduces the pressure within the disc, and can also reduce contact pressure between the displaced nuclear material and neutral elements in some cases $^{29)}$.

Our data do not explain the effect of APTT on the change of the anterior pelvic tilt angle when KT is applied continuously, since the data obtained in this study represent only the immediate effects of taping. Future studies are needed to investigate the change in anterior pelvic tilt angle after a long period of APTT. Additionally, the effect of APTT on the anterior pelvic tilt in patients with a posterior pelvic tilt also needs to be studied.

\section{ACKNOWLEDGMENT}

This work was supported by the 2010 Inje University research grant.

\section{REFERENCES}

1) Sarikaya $S$, Ozdolap $S$, Gümüştaş $S$, et al.: Low back pain and lumbar angles in Turkish coal miners. Am J Ind Med, 2007, 50: 92-96.

2) Levine $D$, Whittle MW: The effects of pelvic movement on lumbar lordosis in the standing position. J Orthop Sports Phys Ther, 1996, 24: 130-135.

3) Neumann DA: Kinegiology of the musculoskeletal system: foundations for physical rehabilitation(1st ed). St Louis: Mosby. 2002.

4) Evcik D, Yücel A: Lumbar lordosis in acute and chronic low back pain patients. Rheumatol Int, 2003, 23: 163-165.

5) Lin RM, Jou IM, Yu CY: Lumbar lordosis: normal adults. J Formos Med Assoc, 1992, 9: 329-333.

6) Harrison DD, Cailliet R, Janik TJ, et al.: Elliptical modeling of the sagittal lumbar lordosis and segmental rotation angles as a method to discriminate between normal and low back pain subjects. J Spinal Disord, 1998, 11: 430 439.

7) Jackson RP, McManus AC: Radiographic analysis of sagittal plane alignment and balance in standing volunteers and patients with low back pain matched for age, sex, and size. A prospective controlled clinical study. Spine, 1994, 19: $1611-1618$

8) O'Sullivan PB, Mitchell T, Bulich P, et al.: The relationship beween posture and back muscle endurance in industrial workers with flexion-related low back pain. Man Ther, 2006, 11: 264-271.

9) Wiggins GC, Ondra SL, Shaffrey CI: Management of iatrogenic flat-back syndrome. Neurosurg Focus, 2003, 15: E8.

10) Lee DG: The Pelvic Girdle: an approach to the examination and treatment of the lumbopelvic-hip region (3rd ed). Edinburgh: Churchill Livingstone, 2004.

11) Tullberg T, Blomberg, S, Branth, B, et al.: Manipulation does not alter the position of the sacroiliac joint: A Roentgen Stereophotogrammetric Analysis. Spine, 1998, 23: 1124-1128.

12) Hsu YH, Chen WY, Lin HC, et al.: The effects of taping on scapular kinematics and muscle performance in baseball players with shoulder impingement syndrome. J Electromyogr Kinesiol, 2009, 19: 1092-1099.

13) Osterhues DJ: The use of Kinesio Taping in the management of traumatic patella dislocation. A case study. Physiother Theor Pract, 2004, 20: 267-270.

14) Christou EA: Patellar taping increases vastus medialis oblique activity in the presence of patellofemoral pain. J Electromyogr Kinesiol, 2004, 14: 495-504.

15) Hinman RS, Crossley KM, McConnell J, et al.: Efficacy of knee tape in the management of osteoarthritis of the knee: blinded randomized controlled trial. BMJ, 2003, 327: 135-140.

16) McConnell J, McIntosh B: The effect of tape on glenohumeral rotation range of motion in elite junior tennis players. Clin J Sport Med, 2009, 19: 90-94.

17) Petrone MR, Guinn J, Reddin A, et al.: The accuracy of the palpation meter(PALM) for measuring pelvic crest height difference and leg length discrepancy, J Orthop Sports Phys Ther, 2003, 33: 319-325.

18) Krawiec CJ, Denegar CR, Hertel J, et al.: Static innominate asymmetry and leg length discrepancy in asymptomatic collegiate athletes. Man Ther, 2003, 8: 207-213.

19) Preece SJ, Willan P, Nester CJ, et al.: Variation in pelvic morphology may prevent the identification of anterior pelvic tilt. J Man Manip Ther, 2008, 16: $113-117$.

20) Gnat R, Saulicz E, Bialy M, et al.: Does pelvic asymmetry always mean pathology? Analysis of mechanical factors leading to the asymmetry. J Human kinetics, 2009, 21: 23-35.

21) Kase K, Wallis J: The latest Kinesio taping method. Tokyo: Ski-Journal, 2002.

22) Vaughn HT, Nitsch W: Ilial anterior rotation hypermobility in a female collegiate tennis player. Phys Ther, 2008, 88: 1578-1590.

23) Kendall FP, McCreary EK, Provance PG, et al.: Muscles: Testing and Function, with Posture and Pain (5th ed). Baltimore: Lippincott Williams \& Wilkins, 2005.

24) Kandel ER, Schwartz JH, Jessell TM: Principles of Neural Science. New York: McGraw-Hill; 1991.

25) Murray H, Husk L: Effect of Kinesio ${ }^{\mathrm{TM}}$ taping on proprioception in the ankle. J Orthop Sports Phys Ther, 2001, 31: A-37.

26) Kase K, Tatsuyuki H, Tomoki O: Development of Kinesio ${ }^{\mathrm{TM}}$ tape. Kinesio ${ }^{\mathrm{TM}}$ Taping Perfect Manual. Tokyo: Kinesio Taping Association, 1996.

27) Yoshida A, Kahanov L: The effect of kinesio taping on lower trunk range of motions. Res Sports Med, 2007, 15: 103-112.

28) Tsuji T, Matsuyama $Y$, Sato K, et al.: Epidemiology of low back pain in the elderly: correlation with lumbar lordosis. J Orthop Sci, 2001, 6: 307-311.

29) Magnusson ML, Aleksiev AR, Spratt KF, et al.: Hyperextension and spine height changes. Spine, 1996, 21: 2670-2675. 